\title{
A three-state model for the Photo-Fries rearrangement
}

\author{
Josene M. Toldo, ${ }^{a, b}$ Mario Barbatti ${ }^{b}$ and Paulo F. B. Gonçalves ${ }^{a}$
}

\begin{abstract}
A three-state model for the Photo-Fries rearrangement (PFR) is proposed based on multiconfigurational calculations. It provides a comprehensive mechanistic picture of all steps of the reaction, from the photoabsorption to the final tautomerization. The three states participating in the PFR are an aromatic ${ }^{1} \pi \pi^{*}$, which absorbs the radiation; a predissociative ${ }^{1} n \pi^{*}$, which transfers the energy to the dissociative region; and a ${ }^{1} \pi \sigma^{*}$, along which dissociation occurs. The transfer from ${ }^{1} \pi \pi^{*}$ to ${ }^{1} n \pi^{*}$ involves pyramidalization of the carbonyl carbon, while transfer from ${ }^{1} n \pi^{*}$ to ${ }^{1} \pi \sigma^{*}$ takes place through $\mathrm{CO}$ stretching. Different products are available after a conical intersection with the ground state. Among them, a recombined radical intermediate, which can yield ortho-PFR products after an intramolecular 1,3-H tunneling. The threestate model is developed for phenyl acetate, the basic prototype for PFR, and it reconciles theory with a series of observations from time-resolved spectroscopy. It also delivers a rational way to optimize PFR yields, since, as shown for four different systems, diverse substituents can change the energetic order of the ${ }^{1} \pi \pi^{*}$ and ${ }^{1} n \pi^{*}$ states, preventing or enhancing PFR.
\end{abstract}

\section{Introduction}

Photo-Fries rearrangement (PFR) - a photochemical conversion of aryl esters to ortho- and para-hydroxyphenones (Scheme 1)-is a key step in the synthesis of a large number of compounds. ${ }^{1-4}$ It also plays an important role in the design of functional polymers ${ }^{5-8}$ and in the photodegradation of drugs ${ }^{9,10}$ and agrochemicals. ${ }^{11-13}$ Compared to its thermal version, the Lewis-acid catalyzed Fries rearrangement, PFR has an additional benefit of being a greener synthetic route, since it can be achieved under milder conditions. ${ }^{3}, 14,15$ Given its importance for synthesis, it is not surprising that PFR has been the subject of numerous investigations in the past. ${ }^{15-36}$ Nevertheless, the conceptual theoretical knowledge of this reaction is still incipient ${ }^{36-}$ 38 and, as we shall see, even the full set of electronic states involved in the reaction has not been yet identified.

Experimental observations have established that PFR takes place in the lowest singlet state $\left(\mathrm{S}_{1}\right)^{36,37}$ although, in some cases, a contribution from upper triplet states is also expected. ${ }^{34,}$, $39-41$ The homolytic cleavage of the $\mathrm{OC}-\mathrm{O}$ bond gives rise to a carbonyl and phenoxyl radical pair. The subsequent recombination leads to the starting ester and to cyclohexadienone intermediate, which tautomerizes to yield the rearranged products. The final step is a hydrogen shift, which can proceed either via tunneling or through solvent rearrangement. ${ }^{42,}{ }^{43}$ Alternatively, the radicals can escape from the solvent cage leading to formation of the corresponding

a. Department of Physical Chemistry, Federal University of Rio Grande do Sul, Av. Bento Gonçalves, 9500, Porto Alegre-RS, CEP 91501-970, Brazil.

b. Aix Marseille Univ, CNRS, ICR, Marseille, France.

*Corresponding authors : josene.toldo@ufrgs.br (JMT), mario.barbatti@univamu.fr (MB), paulo@iq.ufrgs.br (PFBG).

Electronic Supplementary Information (ESI) available: Molecular orbitals, absorption spectra, reaction path, energies, and Cartesian coordinates. See DOI: $10.1039 / x 0 \times x 00000 x$ phenol. In addition, the reaction quantum yield of rearranged products is strongly influenced by solvent polarity as well as by the presence of electron donor or acceptor in the aromatic moiety. ${ }^{19,27,}$ $32,44,45$

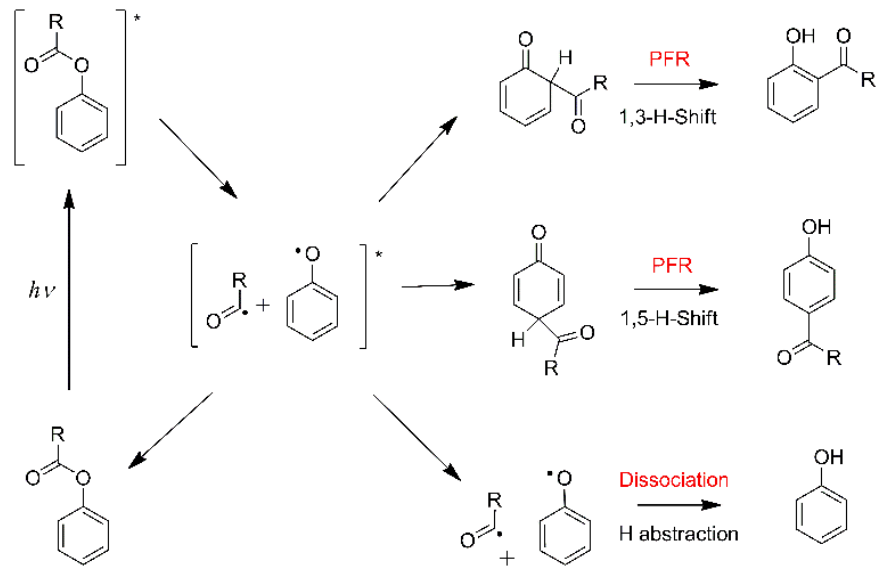

Scheme 1. General scheme of Photo-Fries rearrangement. For pheny acetate, $\mathrm{R}=$ methyl.

Further insights into the early events in PFR of phenyl acetate (PA) in cyclohexane has been provided using transient electronic and vibrational absorption spectroscopies. Pumping at $267 \mathrm{~nm}$, they show radical pairs being formed within 28 ps, although phenoxyl radicals are observed as early as 15 ps. $^{36}$ Two-color femtosecond pump-probe spectroscopy pumped at $258 \mathrm{~nm}$, revealed that the $\mathrm{S}_{1}$ state of para-tBu-PA, also in cyclohexane, is depopulated via ${ }^{1} \pi \sigma^{*}$ within just $2 \mathrm{ps}$ and the dissociated radicals recombine within $13 \mathrm{ps} .{ }^{37}$

In contrast to a large number of experimental studies, the last theoretical investigation on PFR was delivered by Grimme, in 1992, 
using semi-empirical methods. ${ }^{38}$ In that work, barriers between 0.9 and $1.2 \mathrm{eV}$ were found for PA photodissociation starting from a ${ }^{1} n \pi$ * state. Such large barriers are clearly incompatible with the measured picosecond time scale of the process. ${ }^{36,37}$ Moreover, still due to methodological limitations of that early work, ${ }^{38}$ the relative importance of dissociation along ${ }^{1} n \pi *$ versus ${ }^{1} \pi \sigma^{*}$ could not be clearly stated. In fact, the lack of high-level theoretical information on PFR is such that even the character of the initial excited state${ }^{1} n \pi^{*}$ or ${ }^{1} \pi \pi^{*}$-has still been under debate. ${ }^{36-38}$

Given the knowledge gap between theory ${ }^{38}$ and the most recent experimental works, ${ }^{36}, 37$ our aim has been to provide a comprehensive picture of PFR, based on high-level multiconfigurational theoretical methods, applied to PA in the gas phase, the minimum prototype to understand PFR. The multiconfigurational theoretical approach has allowed us to clarify several the following questions: Which state is initially populated? How is the energy transferred from the Franck-Condon region to the dissociative pathway? What are the electronic states involved and their multiplicities during dissociation? Is there any relevant conical intersection along the way? Why does the photoexcited population branches into dissociated and rearranged species? How does tautomerization occur?

Our results revealed that PFR involves three electronic excited states arranged along a specific topography that allows transferring the photoenergy from the aromatic to the carbonyl region. To further explore this three-state model for PFR, we extended the calculations for three other aromatic esters containing an amino group instead of a methyl group attached to carbonyl moiety. As result, we succeed in providing a solid conceptual basis for a class of reactions important for organic, polymer, and environmental chemistry, answering questions that have hindered progress in these fields and laying the groundwork for interpreting four decades of experiments.

\section{Computational details}

Theoretical calculations were carried out using MS-CASPT2//CASSCF protocol, ${ }^{46}$ in which energies are computed at the multi-state complete active space second-order perturbation theory (MS-CASPT2) on structures optimized at the complete active space self-consistent field level (CASSCF). Critical points (minima, transition states, and conical intersection) and reaction paths were optimized with an active space including 14 electrons in 12 orbitals and state-averaged over three states (SA3-CASSCF(14,12)). Cartesian coordinates for all these structures are given in the Supporting Information (SI). The active space for PA was composed of seven occupied and five virtual orbitals: $4 \pi$ and $4 \pi^{*}$, 1 orbital pair $\sigma / \sigma^{*}$ along the OC-O bond, and two non-bonded electrons pairs, one in the oxygen of the carbonyl group and another in the oxygen bonded to the phenyl ring (see SI1). This active space was kept during the subsequent geometry optimizations. For the remaining molecules investigated-phenyl carbamate, metoxyphenyl carbamate, and 2-isopropoxyphenyl Nmethylcarbamate-the active space included the same set of orbitals as described for PA. For the description of the excited states, the CASSCF was still averaged over three states, whereas the energy was corrected with MS-CASPT2 over 7 states. In the CASPT2 calculations, the standard IPEA parameter ${ }^{47}$ of 0.25 a.u. was used and an imaginary level shift ${ }^{48}$ of 0.1 a.u was applied to deal with intruder states. The ANO-S-VDZP49 basis set was employed in all calculations. The $S_{1} / S_{0}$ conical intersection was initially optimized at the CASSCF level. Due to the usual energy split when CASPT2 is computed for such geometries, ${ }^{50}$ the intersection was further relaxed at CASPT2 level. Thus, starting from the CASSCF geometry, restricted optimizations along the $\mathrm{CO}-\mathrm{O}$ bond $(\mathrm{R})$ were done at the MS3-CASPT2 $(6,6)$ level, until the $S_{1}$ and $S_{0}$ states became degenerated. For this final intersection geometry, energies were computed at MS3-CASPT2 $(14,12)$. The subsequent pathway after the $\mathrm{Cl}$ was optimized in the ground state at the CASSCF level, still along constrained values of $\mathrm{R}$. The branch yielding the radical pair was calculated starting from large values of $R$, while the branch giving rise to PFR was calculated systematically increasing R starting from the $\mathrm{Cl}$ structure. All calculations were carried out using MOLCAS 8 program. ${ }^{51}$

\section{Results and discussions}

\section{The PFR mechanism}

The analysis of relaxed reaction pathways in the excited states computed with MS-CASPT2//CASSCF shows that after photoexcitation, PFR takes place through the $S_{1}$ state involving three diabatic characters. A schematic potential energy profile summarizing this three-state model is shown in Figure 1 for PA. Along the solid lines, the OC-O bond distance is the main reaction coordinate, while along the dashed curve, the hydrogen shift between the oxygen and ortho carbon is the main reaction coordinate. Although the relative energies in this figure correspond to those for PA, this three-sates profile is still valid for other molecules undergoing PFR, as discussed later.

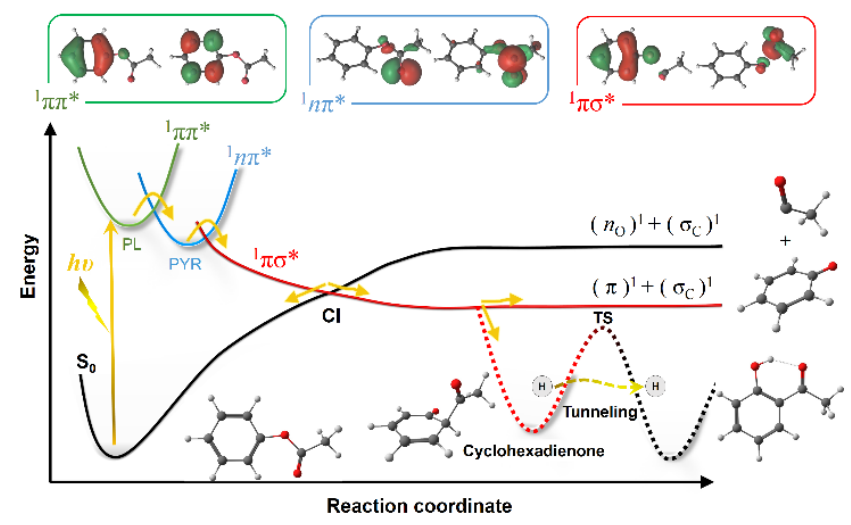

Figure 1. Schematic overview of the three-state model for PFR applied to PA. The insets show the main orbital transitions of the states involved in the PFR. Along the solid lines, the OC-O bond distance is the main reaction coordinate. Along the dashed curve, the hydrogen shift between the oxygen and ortho carbon is the main reaction coordinate. 
Table 1. Vertcalexctain energy $\left(\Delta E_{\text {vert }}\right)$ from the ground state minimum, oscillator strength $\left(f_{o s c}\right)$, and main configuration of the lowest excited states calculated using MS-CASPT2//CASSCF.

\begin{tabular}{lllc}
\hline State & $\Delta E_{\text {vert }}(\mathrm{eV})$ & $f_{\text {osc }}$ & Configuration \\
\hline $\mathrm{S}_{1}$ & 4.82 & 0.0028 & ${ }^{1} \pi \pi *$ \\
$\mathrm{~S}_{\mathbf{2}}$ & 5.86 & 0.1746 & ${ }^{1} \pi \pi *$ \\
$\mathrm{~S}_{3}$ & 6.03 & 0.0020 & ${ }^{1} n \pi *$ \\
\hline
\end{tabular}

In the Franck-Condon region, the $\mathrm{S}_{1}$ state has a dark ${ }^{1} \pi \pi^{*}$ character (Table 1; see SI2 too). A second ${ }^{1} \pi \pi^{*}$ state with larger oscillator strength appears with energy close to the ${ }^{1} n \pi *$ state, but about $1 \mathrm{eV}$ higher than $\mathrm{S}_{1}$. Thus, pumping PA at $267 \mathrm{~nm}(4.64 \mathrm{eV})$, as done in Ref. ${ }^{36}$, excites the dark ${ }^{1} \pi \pi^{*}$ state. Note yet that while the excited electronic density of the ${ }^{1} \pi \pi^{*}$ states are located on the aromatic ring, that of the ${ }^{1} n \pi^{*}$ is mainly at the acetyl moiety (see molecular orbitals in Figure 1).

During the optimization of $\mathrm{S}_{1}$, two minima were found (see Figure 2). The first one $\left(\mathrm{S}_{1}-\mathrm{PL}\right)$ has a $C_{s}$ geometry with a planar conformation of the acetyl moiety. It features a ${ }^{1} \pi \pi^{*}$ character still located on the aromatic ring. The second minimum ( $S_{1}-P Y R$ ) has a significant pyramidalization of the carbon atom on the acetyl moiety, displacing the oxygen out of the molecular plane. It features a ${ }^{1} n \pi^{*}$ character located in the acetyl moiety only. The $\mathrm{S}_{1}$-PYR minimum is $0.12 \mathrm{eV}$ below the $\mathrm{S}_{1}-\mathrm{PL}$ minimum. A linear interpolation in internal coordinates shows that the barrier to converting between them is smaller than $0.38 \mathrm{eV}$ (shown in $\mathrm{SI3}$ ).

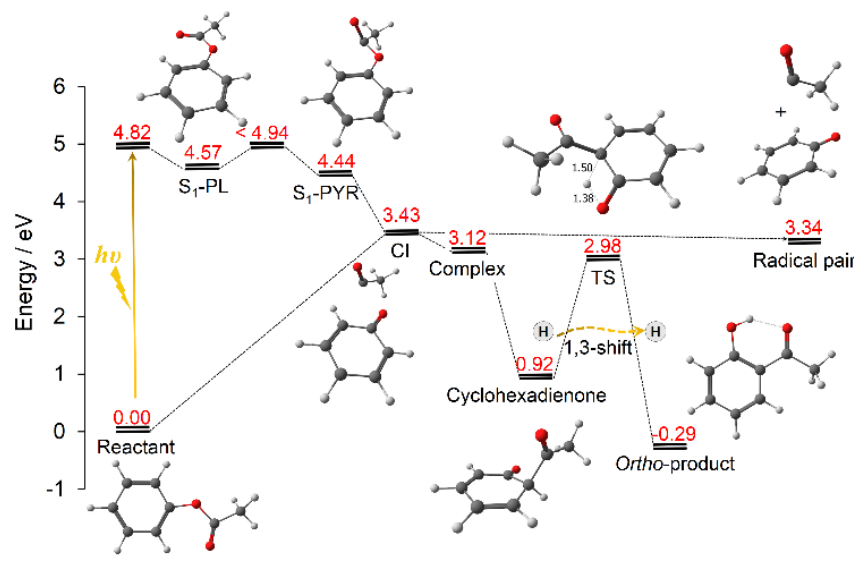

Figure 2. MS-CASPT2 energies long the Photo-Fries rearrangement of PA. CI: Conical intersection; TS: Transition state.

According to previous works, photodissociation in PFR is mediated by a higher electronically excited state with ${ }^{1} \sigma \pi^{*}$ character. ${ }^{36-38}$ It can be seen in Figure 3 that when the acetyl moiety is shifted along the reaction coordinate, this state stabilizes, till becoming $S_{0}$. Due to the uncertainty pointed out in previous works about the assignment of the electronic configuration of the $\mathrm{S}_{1},{ }^{36}$ both $1 \pi \pi^{*}$ and ${ }^{1} n \pi^{*}$ states were investigated as starting point for the OC-O bond breaking. The $\mathrm{S}_{1}$-relaxed potential energy profiles in Figure 3 show that the dissociation is only possible after accessing the ${ }^{1} n \pi^{*}$ state because the barrier to reach the dissociative state starting from
${ }^{1} \pi \pi^{*}$ excited state is exceedingly large, about $0.8 \mathrm{eV}$. On the other hand, no barrier was found when the photodissociation started from the pyramidalized minimum in the ${ }^{1} n \pi^{*}$ state. As result, pyramidalization of the acetyl moiety is required for photodissociation and, in this way, the ${ }^{1} n \pi^{*}$ state can be considered as a pre-dissociative state.

The small barrier between ${ }^{1} \pi \pi^{*}$ and ${ }^{1} n \pi^{*}(<0.38 \mathrm{eV})$ and the barrierless transition from ${ }^{1} n \pi^{*}$ to ${ }^{1} \pi \sigma^{*}$ is the key to understanding how the ${ }^{1} \pi \sigma^{*}$ can be populated in just 2 ps, as experimentally observed. ${ }^{37}$ We mentioned above that dissociation barriers between 0.9 and $1.2 \mathrm{eV}$ were predicted for PA in an early theoretical work. ${ }^{38}$ Such extremely large barriers arose from the geometric constraints imposed in that study, which carried out a limited exploration of the dihedral angle and did not consider pyramidalization at all.

A conical intersection between the dissociative ${ }^{1} \pi \sigma^{*}$ and the $S_{0}$ surfaces is indicated in Figure 3 close to $2.2 \AA$. This crossing is a common feature for $\mathrm{PA}^{38}$ and related molecules, as phenol ${ }^{52}$ and para-tBut-phenyl acetate. ${ }^{37} \mathrm{After}$ the intersection, the reaction path splits in three ways, with one branch returning to the $S_{0}$ parent molecule, another branch following the ${ }^{1} \pi \sigma^{*}$ state originating a cyclohexanone-acetyl radical pair, and a third branch forming a stable cyclohexadienone intermediate.

The population of these three branches is the key step for the PFR yield and it should depend on the particular excited-state topography for each molecule and on the solvent as well. If the conical intersection is reached with a high excess of kinetic energy, radical-pair formation will dominate. But even in this case, PFR can still take place as the solvent cage ${ }^{53}$ may inhibit dissociation and induce recombination of the radical into substituted 2,4- and 2,5cyclohexadienone. In cyclohexane, the formation of cyclohexanone intermediate is found to occur between $13 \mathrm{ps}^{37}$ (para-tBut-PA excited at $258 \mathrm{~nm}$ ) and $42 \mathrm{ps}^{36}(\mathrm{PA}, 267 \mathrm{~nm})$. In this latter case, the fraction of radical pairs formed that are expected to escape from the solvent cage is about $26 \%$ and most of the recombination products (54\%) will be $\mathrm{S}_{0}$ parent molecules. ${ }^{36}$

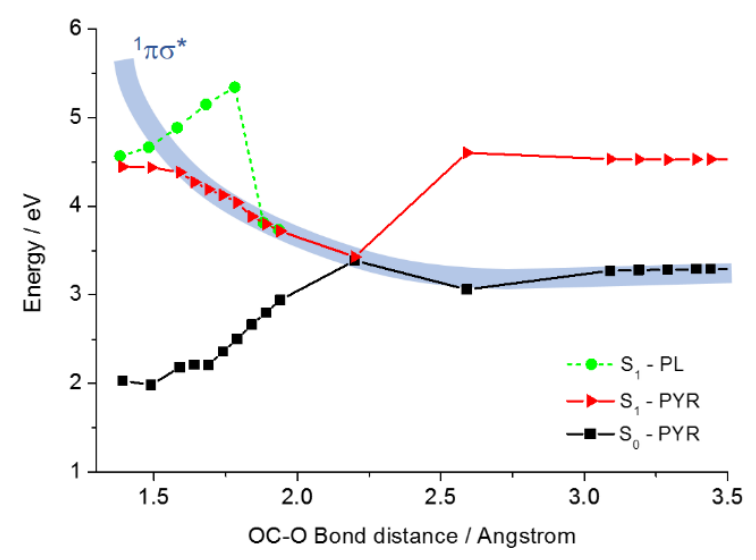

Figure 3. MS-CASPT2 relaxed energy profile starting from the pyramidalized $\left({ }^{1} n \pi^{*}\right) S_{1}$ minimum (solid lines). The $S_{1}$ energy profile starting from the planar $\left({ }^{1} \pi \pi^{*}\right) S_{1}$ minimum is also shown with a dashed line. The shaded curve indicates the ${ }^{1} \pi \sigma^{*}$ state. 
The last step of PFR is enolization of the cyclohexadienone intermediate to form the final substituted hydroxyphenone product. In the gas phase and in nonpolar aprotic solvents, $\mathrm{H}$-shift is intramolecular. As shown in Figure 2, the energy barrier for $1,3-\mathrm{H}$ shift in the gas phase is $2.1 \mathrm{eV}$ and can only be crossed via tunneling. ${ }^{36,} 42$ In methylcyclohexane at $293 \mathrm{~K}$, for instance, the measured tunneling rate for the $1,3-\mathrm{H}$ shift is $3.6 \mathrm{~s}^{-1.42}$ In protic solvents, as alcohols, the $1,3-\mathrm{H}$ and $1,5-\mathrm{H}$ shifts should be much faster, as they are aided by intermolecular interactions. ${ }^{42}$

Table 1. Energy differences between the lowest singlet state and the lowest triplet states calculated at the $\mathrm{S}_{1}-\mathrm{PL}$ and $\mathrm{S}_{1}-\mathrm{PYR}$ minima using MS-CASPT2//CASSCF. The main configuration of the triplet states is shown as well.

\begin{tabular}{lllll}
\hline State & $\Delta E(\mathrm{eV})$ & Config. & $\Delta E(\mathrm{eV})$ & Config. \\
& $\mathrm{S}_{1-\mathrm{PL}^{-} \mathrm{T}_{\mathrm{X}}{ }^{[\mathrm{a}]}}$ & & $\mathrm{S}_{1-\mathrm{PYR}-\mathrm{T}_{\mathrm{X}}{ }^{[\mathrm{b}]}}$ & \\
\hline $\mathrm{T}_{1}$ & -0.71 & ${ }^{3}\left(\pi \pi^{*}\right)$ & -0.19 & ${ }^{3}\left(n \pi^{*}\right)_{\mathrm{CO}}$ \\
$\mathrm{T}_{2}$ & -0.09 & ${ }^{3}\left(\pi \pi^{*}\right)$ & 0.44 & ${ }^{3}\left(\pi \pi^{*}\right)$ \\
$\mathrm{T}_{3}$ & -0.02 & ${ }^{3}\left(\pi \pi^{*}\right)$ & 1.71 & ${ }^{3}\left(\pi \pi^{*}\right)$ \\
\hline
\end{tabular}

[a] Relative to the $S_{1}-P L$ optimized geometry.

[b] Relative to the $S_{1}$ - PYR optimized geometry.

Triplet states around the ${ }^{1} \pi \pi^{*}$ and ${ }^{1} n \pi^{*}$ minima were also calculated to ascertain the spin multiplicity of the photodissociation process. Table 2 shows the energy of the triplet states compared to the energy of the lowest singlet state in $\mathrm{S}_{1-}$ planar $\left({ }^{1} \pi \pi^{*}\right)$ and $S_{1}$-pyramidalized $\left({ }^{1} n \pi^{*}\right)$ geometries. There are two triplet states near the $S_{1}$ state at the ${ }^{1} \pi \pi^{*}$ minimum, but they both have ${ }^{3} \pi \pi^{*}$ character and thus intersystem crossing to them should be negligible according to El-Sayed rules. ${ }^{54,5}$ Similarly, there is a triplet state near the $S_{1}$ state at the ${ }^{1} n \pi^{*}$ minimum, but it has ${ }^{3} n \pi^{*}$ character and intersystem crossing to it will be negligible for the same reason. Therefore, in the case of PA, the photodissociation proceeds via $S_{1}$, explaining the experimental results. ${ }^{36,37}$

\section{PFR sensitivity to substituents}

Besides phenyl acetate, the three-state model for PFR was applied to three other molecules to demonstrate the utility of this model to rationalize this type of reaction. In these additional molecules (see Figure 4-top), the methyl group attached to carbonyl moiety was replaced by an amino group, $\mathrm{NH}_{2}$ (in $\mathbf{b}$ and $\mathbf{c}$ ) and $\mathrm{NHCH}_{3}$ in $\mathbf{d}$, which results in a carbamate group. In molecules $\mathbf{c}$ and $\mathbf{d}$, an electron donor group was attached to the ortho position $\left(\mathrm{OCH}_{3}\right.$ and $\mathrm{OCH}\left(\mathrm{CH}_{3}\right)_{2}$, respectively). The largest of these molecules (d), known as Propoxur (or commercially as Baygon), is an important pesticide, for which its photochemistry holds a major practical interest as a key to determine its fate in the environment.

CASPT2//CASSCF results for the $S_{1}$ minima show that changing the methyl by an amino group inverts the energetic order of the planar $\left({ }^{1} \pi \pi^{*}\right)$ and pyramidal $\left({ }^{1} n \pi^{*}\right)$ minima (see Figure 4$)$. While with the methyl group, the ${ }^{1} n \pi^{*}$ state is more stable than the ${ }^{1} \pi \pi^{*}$ by $0.1 \mathrm{eV}$, with the amino group, the ${ }^{1} \pi \pi^{*}$ becomes the most stable by 0.2
$\mathrm{eV}$ in $\mathbf{b}$ and by $0.4 \mathrm{eV}$ in $\mathbf{c}$ and $\mathbf{d}$. We can rationalize this effect based on the resonance structures that characterize the amino-substituted molecules, stabilizing the lone pairs and increasing the energies of $\mathrm{n} \pi^{*}$ state. We note, however, that the correct description of the ${ }^{1} \pi \pi^{*}{ }_{-}^{1} n \pi^{*}$ energy gap requires a proper account of electron dynamic correlation, as revealed by the strong differences between CASSCF and MS-CASPT2 results (SI4).

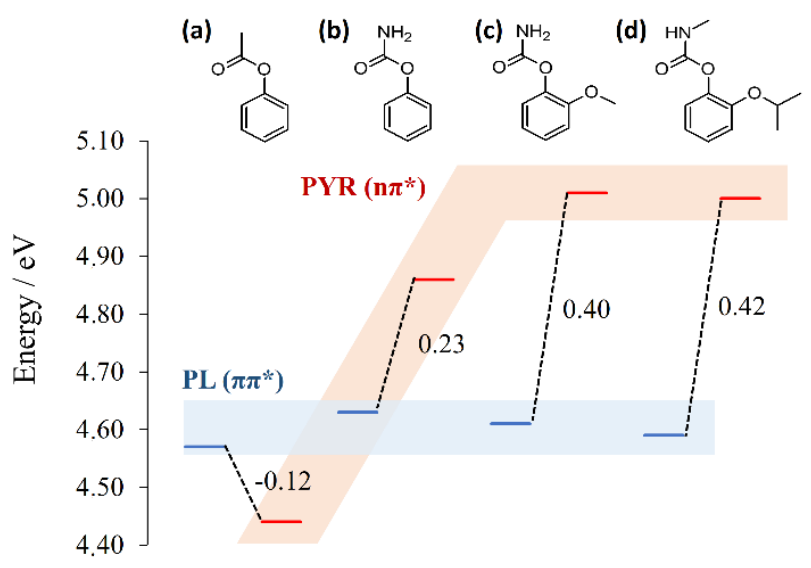

Figure 4. Energies of the planar ${ }^{1}\left(\pi \pi^{*}\right)$ and pyramidal ${ }^{1}\left(n \pi^{*}\right) S_{1}$ minima of (a) phenyl acetate, (b) phenyl carbamate, (c) orthomethoxyphenyl carbamate, and (d) 2-isopropoxyphenyl methylcarbamate (Propoxur) in the gas phase. Computed with CASPT2//CASSCF.

As discussed, the three-state model predicts that populating the ${ }^{1} n \pi^{*}$ state is a requisite to reach the dissociative ${ }^{1} \pi \sigma^{*}$ state. Therefore, we may conclude that in molecules $\mathbf{b}$ to $\mathbf{d}$, PFR is unfavorable in the gas phase. The relative energy between the two $\mathrm{S}_{1}$ minima helps to understand why Propoxur (d) diluted in different organic solvents does not undergo PFR, while it does in water. ${ }^{56,57} \mathrm{As}$ it can be seen in Figure 4 , for all molecules, the ${ }^{1} \pi \pi^{*}$ state has approximately the same energy, while the ${ }^{1} n \pi^{*}$ state is strongly destabilized by changing the methyl by an amino group. Thus, while in organic solvents, Propoxur behaves essentially as in the gas phase, with ${ }^{1} n \pi^{*}>1 \pi \pi^{*}$ (PFR unfavorable), in water, the interaction with the water oxygen disrupts the O-N correlation between $n$ electrons, causing ${ }^{1} \pi \pi^{*}>{ }^{1} n \pi^{*}$ (PFR favorable). ${ }^{56,57}$

Summarizing, the energetic balance between the ${ }^{1} \pi \pi^{*},{ }^{1} n \pi^{*}$, and ${ }^{1} \pi \sigma^{*}$ states is critical for PFR (Figure 1). Depending on the substituents (or solvent) the ${ }^{1} \pi \pi^{*}$ can be stabilized relative to the ${ }^{1} n \pi^{*}$ state, which leads to an overall reduction of PFR yield and an increase of luminescence yield. Another substituent (or solvent) stabilizing the ${ }^{1} n \pi^{*}$ relative to the ${ }^{1} \pi \sigma^{*}$ would lead to an increase of PFR yield and reduction of radical pair production. Thus, the threestate model for PFR can be applied to engineering compounds aiming at maximizing specific products and to rationalize experimentally observed outputs. 


\section{Conclusions}

Our theoretical analysis provides a clear and comprehensive picture for PFR. We have shown that the bond cleavage is due to an interplay of three singlet electronic states: an aromatic ${ }^{1} \pi \pi^{*}$, which absorbs the radiation and it is initially populated; a carbonyl ${ }^{1} n \pi^{*}$, which transfers the electronic energy from the aromatic ring to the dissociative region; and a ${ }^{1} \sigma \pi^{*}$, responsible for the homolytic cleavage. For phenyl acetate, the transfers between these three states occur with a small barrier between ${ }^{1} \pi \pi^{*}$ and ${ }^{1} n \pi^{*}$, and without any significant barrier between ${ }^{1} n \pi^{*}$ and ${ }^{1} \sigma \pi^{*}$. Direct transfer from ${ }^{1} \pi \pi^{*}$ to ${ }^{1} \sigma \pi^{*}$ is precluded by large energy barriers. Triplet dissociation is also not possible due to El-Sayed rules. After transferring to ${ }^{1} \sigma \pi^{*}$ and reaching a conical intersection, the molecule may return to the parent species, dissociate, or form cyclohexadienone intermediates, which are precursors for PFR. In the gas phase and in nonpolar aprotic solvents, the orthosubstituted product is obtained after slow hydrogen tunneling, while in protic solvents, intermolecular $\mathrm{H}$ shift should dominate.

The three-state model for PFR provides a general picture beyond the PA prototype, as the photodissociation process in phenyl acetate should be analogous to that in other aromatic esters, amides, carbamates, and carbonates. ${ }^{58-60}$ Different substituents and solvents will naturally change the relative energies of the ${ }^{1} \pi \pi^{*},{ }^{1} n \pi^{*}$, and ${ }^{1} \sigma \pi^{*}$ states, quantitatively altering the basic topography illustrated in Figure 1, and leading to different rates and product yields. We have demonstrated, for instance, that in the case of carbamate derivatives, PFR is unfavorable in the gas phase (and likely in nonpolar aprotic solvents too), because the predissociative state $\left({ }^{1} n \pi^{*}\right)$ is too high in energy. Thus, these three states will always ultimately control absorption, energy transfer, and dissociation steps in PFR.

\section{Acknowledgements}

The authors thank CNPq and CAPES (PDSE program, 88881.131599/2016-01) for scholarships and the support from CESUP/UFRGS and Santos Dumont/LNCC, all funded by the Brazilian government. The authors also thank the support of the A*MIDEX grant (ANR-11-IDEX-0001-02) and the project Equip@Meso (ANR-10-EQPX-29-01), both funded by the French Government "Investissements d'Avenirßprogram.This work was performed using HPC resources from GENCI-CINES (Grant 2017A0010810012).

\section{Nołẹs and refferenges, Tetrahedron Lett., 2001, 42, 7193.}

2. W. Bowers, T. Ohta, J. Cleere and P. Marsella, Science, 1976, 193, 542.

3. F. Galindo, M. C. Jiménez and M. A. Miranda, in Arene chemistry: Reaction mechanism and methods for aromatic compounds, ed. J. Mortier, John Wiley \& Sons, 2016.

4. T. Magauer, H. J. Martin and J. Mulzer, Angew. Chem. Int. Ed., 2009, 48, 6032.

5. A. Rivaton, B. Mailhot, J. Soulestin, H. Varghese and J. L. Gardette, Polym. Degrad. Stab., 2002, 75, 17.
6. T. Höfler, T. Grießer, X. Gstrein, G. Trimmel, G. Jakopic and W. Kern, Polymer, 2007, 48, 1930.

7. T. Höfler, T. Grießer, M. Gruber, G. Jakopic, G. Trimmel and W. Kern, Macromol. Chem. Phys., 2008, 209, 488.

8. Y. Ishida, Y. Takeda and A. Kameyama, React. Funct. Polym. 2016, 107, 20.

9. M. Martignac, E. Oliveros, M. T. Maurette, C. Claparols and F. Benoit-Marquie, Photochem. Photobiol. Sci., 2013, 12, 527.

10. A. C. Weedon and D. F. Wong, J. Photochem. Photobiol. A, 1991, 61, 27.

11. A. Sanjuan, G. Aguirre, M. Alvaro, H. Garcia and J. C. Scaiano, Appl. Catal. B, 2000, 25, 257.

12. M. Passananti, M. Lavorgna, M. R. lesce, M. DellaGreca, E. Criscuolo, A. Parrella, M. Isidori and F. Temussi, Env. Sci. Process. Impact, 2014, 16, 823.

13. C. K. Remucal, Env. Sci. Process. Impact, 2014, 16, 628.

14. Z. Wang, in Comprehensive Organic Name Reactions and Reagents, John Wiley \& Sons, Inc., 2010.

15. D. Iguchi, R. Erra-Balsells and S. M. Bonesi, Photochem. Photobiol. Sci., 2016, 15, 105.

16. J. C. Anderson and C. B. Reese, Proc. Chem. Soc., 1960, 217.

17. M. R. Sandner and D. J. Trecker, J. Am. Chem. Soc., 1967, 89, 5725 .

18. M. R. Sandner, E. Hedaya and D. J. Trecker, J. Am. Chem. Soc., 1968, 90, 7249.

19. G. M. Coppinger and E. R. Bell, J. Phys. Chem., 1966, 70, 3479.

20. J. W. Meyer and G. S. Hammond, J. Am. Chem. Soc., 1970, 92, 2187.

21. C. E. Kalmus and D. M. Hercules, Tetrahedron Lett., 1972, 1575.

22. C. E. Kalmus and D. M. Hercules, J. Am. Chem. Soc., 1974, 96, 449.

23. J. W. Meyer and G. S. Hammond, J. Am. Chem. Soc., 1972, 94.

24. H. J. Yoon, S. H. Ko, M. K. Ko and W. K. Chae, Bull. Korean Chem. Soc., 2000, 21, 901.

25. H. Kobsa, J. Org. Chem., 1962, 27, 2293.

26. D. Bellus, P. Hrdlovic and P. Slama, Collect. Czech. Chem. Commun., 1968, 33, 2646.

27. H. J. Hageman, Tetrahedron, 1969, 25, 6015.

28. Schwetli.K, A. Mehlhorn, R. Noack and J. Stumpe, Zeitschrift Fur Chemie, 1974, 14, 116.

29. A. Mehlhorn, B. Schwenzer and K. Schwetlick, Tetrahedron, 1977, 33, 1483 .

30. M. S. Syamala, B. N. Rao and V. Ramamurthy, Tetrahedron, 1988, 44, 7234 .

31. L. S. Kaanumalle, C. L. D. Gibb, B. C. Gibb and V. Ramamurthy, Org. Biomol. Chem., 2007, 5, 236.

32. R. Suau, G. Torres and M. Valpuesta, Tetrahedron Lett., 1995, 36, 1311.

33. A. K. Zarkadis, V. Georgakilas, G. P. Perdikomatis, A. Trifonov, G. G. Gurzadyan, S. Skoulika and M. G. Siskos, Photochem. Photobiol. Sci., 2005, 4, 469.

34. M. Gohdo and M. Wakasa, Chem. Lett., 2010, 39, 106.

35. A. Zanutta, L. Colella, C. Bertarelli and A. Bianco, Optical Materials, 2013, 35, 2283.

36. S. J. Harris, D. Murdock, M. P. Grubb, G. M. Greetham, I. P. Clark, M. Towrie and M. N. R. Ashfold, Chem. Sci., 2014, 5, 707.

37. S. Lochbrunner, M. Zissler, J. Piel, E. Riedle, A. Spiegel and T. Bach, J. Chem. Phys., 2004, 120, 11634.

38. S. Grimme, Chem. Phys., 1992, 163, 313.

39. N. P. Gritsan, Y. P. Tsentalovich, A. V. Yurkovskaya and R. Z. Sagdeev, J. Phys. Chem., 1996, 100, 4448.

40. I. F. Molokov, Y. P. Tsentalovich, A. V. Yurkovskaya and R. Z. Sagdeev, J. Photochem. Photobiol. A, 1997, 110, 159. 
41. M. Gohdo, T. Takamasu and M. Wakasa, Phys. Chem. Chem. Phys., 2011, 13, 755.

42. T. Arai, S. Tobita and H. Shizuka, J. Am. Chem. Soc., 1995, 117, 3968.

43. T. Arai, S. Tobita and H. Shizuka, Chem. Phy. Lett., 1994, 223, 521.

44. D. A. Plank, Tetrahedron Lett., 1968, 9, 5423.

45. J. W. Meyer and G. S. Hammond, J. Am. Chem. Soc., 1972, 94, 2219.

46. J. Finley, P.-Å. Malmqvist, B. O. Roos and L. Serrano-Andrés, Chem. Phys. Lett., 1998, 288, 299.

47. G. Ghigo, B. O. Roos and P.-A. Malmqvist, Chem. Phys. Lett., 2004, 396, 142.

48. N. Forsberg and P.-Å. Malmqvist, Chem. Phys. Lett., 1997, 274, 196.

49. P.-O. Widmark, P.-Å. Malmqvist and B. O. Roos, Theor. Chim. Acta, 1990, 77, 291.

50. G. Zechmann and M. Barbatti, Int. J. Quantum Chem., 2008, 108, 1266.

51. F. Aquilante, J. Autschbach, R. K. Carlson, L. F. Chibotaru, M. G. Delcey, L. De Vico, I. Fdez. Galván, N. Ferré, L. M. Frutos, L. Gagliardi, M. Garavelli, A. Giussani, C. E. Hoyer, G. Li Manni, H. Lischka, D. Ma, P. Å. Malmqvist, T. Müller, A. Nenov, M. Olivucci, T. B. Pedersen, D. Peng, F. Plasser, B. Pritchard, M. Reiher, I. Rivalta, I. Schapiro, J. Segarra-Martí, M. Stenrup, D. G. Truhlar, L. Ungur, A. Valentini, S. Vancoillie, V. Veryazov, V. P. Vysotskiy, O. Weingart, F. Zapata and R. Lindh, J. Comput. Chem., 2016, 37, 506.

52. Z. Lan, W. Domcke, V. Vallet, A. L. Sobolewski and S. Mahapatra, J. Chem. Phys., 2005, 122, 224315.

53. R. Crespo-Otero, A. Mardykov, E. Sanchez-Garcia, W. Sander and M. Barbatti, Phys. Chem. Chem. Phys., 2014, 16, 18877.

54. N. J. Turro, Modern molecular photochemistry, Univ Science Books, 1991.

55. M. A. El-Sayed, J. Chem. Phys., 1963, 38, 2834.

56. G. A. Ana Sanjuán, Mercedes Álvaro, Hermenegildo García, J. C. Scaiano, Appl. Catal. B, 2000, 25, 257.

57. W. Schwack and G. Kopf, Z. Lebensm. Unters. Forsch., 1992, 195, 250.

58. M. A. Miranda and F. Galindo, in Molecular and supramolecular photochemistry: Photochemistry of organic molecules in isotropic and anisotropic media, eds. V. Ramamurthy and K. S. Schanke, Marcel Dekker Inc., New York, 2003, vol. 9, pp. 43.

59. D. J. Trecker, R. S. Foote and C. L. Osborn, Chem. Comm., 1968, 1034.

60. E. A. Caress and I. Rosenberg, J. Org. Chem., 1971, 37, 3160. 